\title{
Adaptação do Índice de Vulnerabilidade Sócio-Climática (ISVC) em uma comunidade rural do semiárido do Brasil
}

\section{Denise Pinto Gadelha ${ }^{1,2}$, Thamires Kelly Nunes Carvalho ${ }^{2}$ e Reinaldo Farias Paiva de Lucena ${ }^{2,3}$}

\author{
${ }^{1}$ Universidade Federal da Paraíba. Centro de Comunicação, Turismo e Artes. \\ Departamento de Turismo e Hotelaria. Campus I. João Pessoa-PB, Brasil \\ (CEP 58051-900). \\ ${ }^{2}$ Universidade Federal da Paraíba. Centro de Ciências Exatas e da Natureza. \\ Programa de Pós-Graduação Em desenvolvimento e Meio Ambiente. Campus I. João \\ Pessoa-PB, Brasil (CEP 58051-900). \\ ${ }^{3}$ Universidade Federal do Mato Grosso do Sul. Instituto de Biociências. Laboratório \\ de Etnobotânica. Campo Grande-MS, Brasil (CEP 79070-900).
}

\begin{abstract}
Resumo. 0 presente estudo tem por objetivo adaptar o Índice de Vulnerabilidade Sócio-Climática (ISVC) para a avaliação de vulnerabilidade de pequenos agricultores residentes no semiárido nordestino. Especificamente, objetiva mapear a estrutura social e cultural da comunidade rural escolhida a partir de indicadores, e que auxiliam no diagnóstico de vulnerabilidade para pequenas localidades. 0 estudo foi desenvolvido no Município de Cabaceiras, Região do Cariri da Paraíba. O índice utilizado tem como base dados retirados do IDH (Índice de Desenvolvimento Humano) municipal, e dados de variáveis climáticas disponíveis no IRCM (Índice Regional de Mudanças Climáticas). A análise estatística foi realizada a partir do software R, e utilizado o pacote "PACMAN". Seguindo o cálculo do IVSC, o resultado obtido para o município de Cabaceiras é IVSC $=5,60$. Esse resultado é o produto da multiplicação do valor obtido para a variável climática (IRMC $=13)$, pela média geométrica do valor obtido para a variável social, que aqui será chamada de Indicadores de Vulnerabilidade Local (IVL $=0,43$ ). 0 Índice de Vulnerabilidade Socio-Climática (IVSC), da forma como foi adaptado e calculado neste estudo, oferece praticidade em sua execução, podendo ser facilmente aplicado para todo o estado da Paraíba e outras regiões do semiárido.
\end{abstract}

Palavras-chave: Caatinga; Mudanças climáticas; Populações tradicionais.

Abstract. Adaptation of the Socio-Climate Vulnerability Index (ISVC) in a rural community in the semiarid region of Brazil. The present study aims to adapt the Socio-Climatic Vulnerability Index (ISVC) to assess the vulnerability of small farmers residing
Recebido

$26 / 12 / 2020$

Aceito

$29 / 04 / 2021$

Publicado

$30 / 04 / 2021$

Acesso aberto

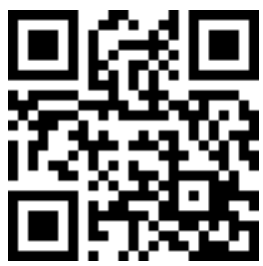

D 0000-0003-1103-265X Denise Pinto Gadelha

(D) 0000-0003-3847-1249 Thamires Kelly Nunes Carvalho 
in the northeast semiarid. Specifically, it aims to map the social and cultural structure of the rural community chosen based on indicators, which assist in the diagnosis of vulnerability for small towns. The study was carried out in the Municipality of Cabaceiras, Region of Cariri da Paraíba. The index used is based on data taken from the municipal HDI (Human Development Index), and data on climate variables available from the RCCI (Regional Climate Change Index). Statistical analysis was performed using the R software, and the "PACMAN" package was used. Following the calculation of the IVSC, the result obtained for the municipality of Cabaceiras is IVSC $=5.60$. This result is the product of multiplying the value obtained for the climate variable (IRMC = 13), by the geometric mean of the value obtained for the social variable, which here will be called Local Vulnerability Indicators (IVL $=0.43$ ). The Socio-Climatic Vulnerability Index (IVSC), as it was adapted and calculated in this study, offers practicality in its execution and can be easily applied to the entire State of Paraíba and other regions of the semiarid region.

Keywords: Caatinga: Climate change; Traditional populations.
D 0000-0002-1195-4315 Reinaldo Farias Paiva de Lucena

\section{Introdução}

A evolução biocultural humana está relacionada com a forma como o homem enxerga e se relaciona com o meio natural, que se reflete na sua forma de vida, cultura, e preferências de manejo de recursos naturais. A forma como o ser humano está ligado ao ambiente natural o coloca em uma condição, muitas vezes, passiva em relação a possíveis episódios fora do padrão esperado e do controle antropogênico.

Os esforços para a compreensão e mapeamento dos locais e populações com maior nível de exposição a ambientes críticos, são cada dia mais impulsionados pelo cenário climático global (IPCC, 2018). Sob esta ótica, espera-se documentar a vulnerabilidade, ou seja, a incapacidade de adaptação a partir do grau de susceptibilidade de um sistema a um dano (McCarthy et al., 2001).

Diversos são os estudos que objetivam diagnosticar a vulnerabilidade climática sob diferentes perspectivas em todo o mundo (Cacioppo et al., 2011; Mainali e Procipe, 2017; Batavia e Nelson, 2018). Em Uganda, Chombo et al. (2018) evidenciam a importância de colocar no centro dos estudos, as populações de agricultores, uma vez que as características biofísicas e socioeconômicas das comunidades rurais as colocam como vulneráveis aos efeitos de extremos climáticos. Argumentam também sobre a necessidade de investigações direcionadas para pequenas localidades.

Com o avanço dos métodos estatísticos, índices e modelos têm sido utilizados para prever atitudes (Beiser-Macgrath e Huber, 2018), moldar mapas de vulnerabilidade de acordo com indicadores socioeconômicos, culturais e biofísicos (Chombo et al., 2018), e prever a vulnerabilidade de espécies vegetais às mudanças climáticas (Butt e Gallagher, 2018), e nesse contexto, Darela-Filho et al. (2016), desenvolveram índice específico para ser utilizado no Brasil. Este índice pode ser adaptado para diferentes tipos de regiões e diferentes escalas territoriais.

Com base no exposto, o presente estudo tem por objetivo adaptar o índice para a avaliação de vulnerabilidade sócio climática de pequenos agricultores na região do Cariri, no semiárido do Estado da Paraíba, Nordeste do Brasil. Especificamente, objetiva mapear a estrutura social e cultural da comunidade rural escolhida a partir de indicadores 
propostos por Gadelha et al. (2021), e que auxiliam no diagnóstico de vulnerabilidade para pequenas localidades, especificamente, comunidades rurais no semiárido do estado da Paraíba.

\section{Metodologia}

\section{Delimitação e caracterização da área de estudo}

0 estudo foi desenvolvido no município de Cabaceiras, região do Cariri da Paraíba (Figura 1). Possui uma área de $452,920 \mathrm{~km}^{2}$, cerca de 5.035 habitantes, concentrados na zona rural (56\%) (IBGE, 2017). Apresenta clima semiárido quente (BSh), com média de temperatura anual de $26{ }^{\circ} \mathrm{C}$, e menor índice de precipitação $(356 \mathrm{~mm})$ anual do país, concentrado entre os meses de fevereiro e maio (CEPED, 2011) (Figura 1).

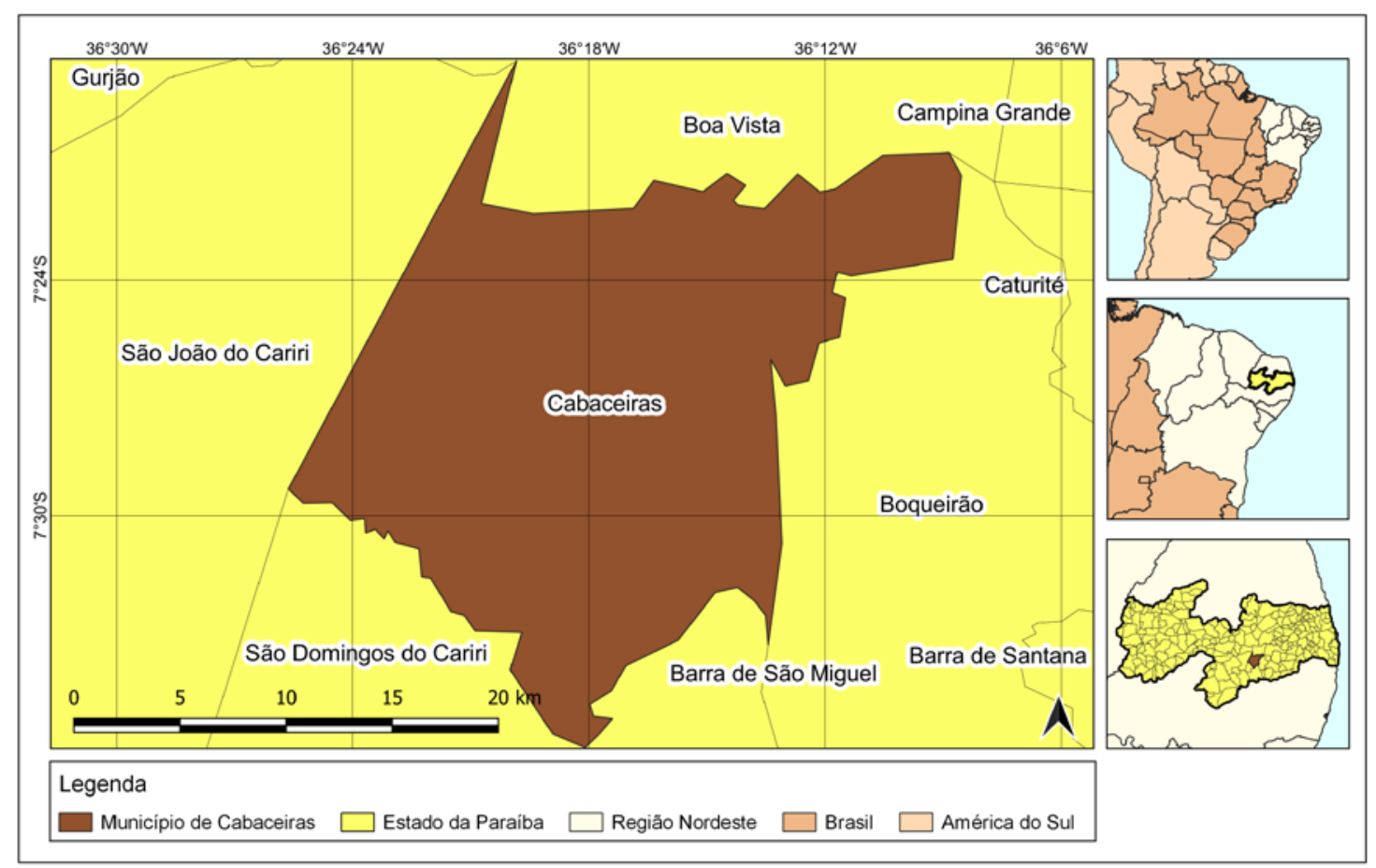

Figura 1. Município de Cabaceiras, Paraíba, Brasil.

\section{Adaptando Índice de Vulnerabilidade Sócio Climática}

Foi utilizado como base para a adaptação o Índice de Vulnerabilidade Sócio-Climática (ISVC) proposto por Torres et al. (2012) e atualizado por Darela Filho et al. (2016) (Figura 2). Este índice demonstra como a intensidade das mudanças climáticas, aliada a outros fatores de desenvolvimento humano, influenciam na vulnerabilidade climática em determinadas regiões, e pode ser aplicado em qualquer escala, desde que se tenha dados disponíveis em uma resolução mínima. 


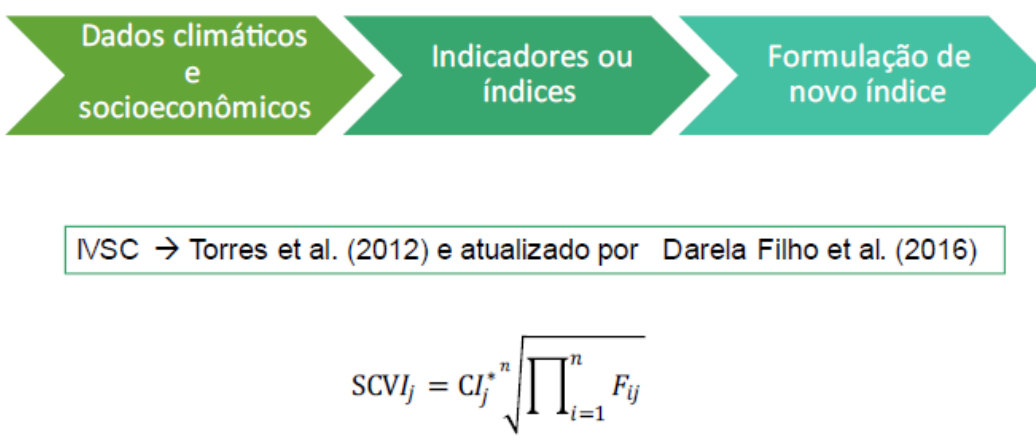

Onde $\circ \mathrm{Cl}$ é representado por qualquer índice que mensure a mudança climática, $i$ a média qeométrica normalizada por indicadores sociais $\left(F_{i}\right)$, calculada para cada grid $j$. Este índice será adaptado para utilização local. Como índice de mudanças climáticas será admitido o RCCI (Regional Climate Change Index - Índice de Mudança Climática Regional), desenvolvido por Giorgi (2006).

Figura 2. Adaptando índice de vulnerabilidade sócio-climática para ser aplicado no estudo na comunidade rural São Francisco, Cabaceiras, Paraíba, Brasil.

A vulnerabilidade descreve um conjunto de condições de uma população que derivam dos contextos cultural, social, ambiental, político e econômico, predominantes e históricos. Neste sentido, o risco a que as populações estão expostas não deriva apenas das alterações climáticas, mas também da sua organização social e padrão de acesso aos recursos.

Este índice tem como base dados retirados do IDH (Índice de Desenvolvimento Humano) municipal, e dados de variáveis climáticas disponíveis no IRCM (Índice Regional de Mudanças Climáticas) que pode ser visto nos estudos desenvolvidos por Giorgi (2006) e Torres e Marengo (2014).

Para a adaptação do IVSC para pequenas localidades, foi utilizada uma base de dados, uma série de indicadores propostos por Gadelha et al. (2021) e que foram normalizados, ou seja, transformados em números que vão de uma escala de 0,1 , e utilizados como substitutos do IDH na equação. Na Tabela 1 temos as variáveis de indicadores utilizadas conforme literatura. Os dados climáticos utilizados nesse trabalho, da mesma forma que Darela Filho et al. (2016), foram retirados do KMNI Climate Change Atlas.

Os indicadores propostos por Gadelha et al. (2021) tem o objetivo de ser a representação da realidade de pequenas comunidades rurais da região semiárida do estado da Paraíba. Os autores supracitados, desenvolveram esses indicadores baseados na Comunidade São Francisco, que fica localizada no município de Cabaceiras (Paraíba, Nordeste do Brasil), por isso, neste estudo, o IVSC será adaptado para calcular a vulnerabilidade sócio-climática dessa região, baseando-se na caracterização da realidade encontrada na zona rural, quanto aos extremos climáticos. 
Tabela 1. Apresentação das variáveis utilizadas com base nos indicadores propostos nesse estudo, e a relação positiva ou negativa quanto a vulnerabilidade. Estudo desenvolvido na comunidade São Francisco em Cabaceiras, Paraíba, Brasil.

\begin{tabular}{|c|l|l|c|}
\hline \multicolumn{1}{|c|}{ Sigla } & \multicolumn{1}{|c|}{ Variáveis } & $\begin{array}{c}\text { Relação positiva ou } \\
\text { negativa }\end{array}$ \\
\hline 1 & ES & Idade (Chefe da casa) & - \\
\hline 2 & D & Escolaridade & + \\
\hline 3 & NHC & Número de habitantes da casa & + \\
\hline 4 & CNJID & Idade do Cônjuge & - \\
\hline 5 & CNJES & Escolaridade do Cônjuge & + \\
\hline 6 & NF & Número de filhos & + \\
\hline 7 & NFD & Número de filiados desempregados & + \\
\hline 8 & NFS & Número de filiados em serviço & - \\
\hline 9 & RPC & Renda per capta & - \\
\hline 101 & PB & Posse de bens & + \\
\hline 11 & AUFS & Acesso à zona urbana (frequência semanal) & - \\
\hline 12 & DAI & Diversidade de acesso à informação & - \\
\hline 13 & AS & Acesso à saúde & - \\
\hline 14 & PA & Participação em associação ou cooperativa & - \\
\hline 15 & VASFM & Visita do agente de saúde (frequência mensal) & - \\
\hline 16 & APT & Acesso a palestras ou treinamento & + \\
\hline 17 & AAPI & Acesso a auxílios para períodos improdutivos & - \\
\hline 18 & PPUS & Perda de plantio na última seca & - \\
\hline 19 & ASE & Acesso a sementes para plantio & + \\
\hline 20 & CRA & Criação atual de rebanho de animais & - \\
\hline 21 & MAS & Morte/venda de animais na última seca & + \\
\hline 22 & FAAC & Fonte de abastecimento de água da casa & - \\
\hline 23 & PEVCN & Perda de espécies vegetais frutíferas na última seca & + \\
\hline 24 & NC & (complementação nutricional) & + \\
\hline 25 & OFTA & Ocasião de total falta de água na residência & - \\
\hline
\end{tabular}

Quanto às variáveis climáticas utilizadas, foram baseadas em Torres e Marengo (2014), que consideraram: [1] alteração de temperatura média na superfície da região relativamente à alteração média global de temperatura, [2] alteração na média regional de precipitação $(\Delta \mathrm{P}, \%$ do valor simulado), [3] alterações na variabilidade interanual de precipitações ( $\Delta \sigma \mathrm{P}, \%$ do valor simulado), [4] e temperatura ( $\Delta \sigma \mathrm{T}, \%$ do valor simulado). Os valores em porcentagem são referentes a diferença entre as variáveis simuladas para o período de 1969-1990 e as variáveis projetadas para o período de 2071-2100.

Para se obter o Índice de Vulnerabilidade Sócio Climática para pequenas localidades, foram seguidos os seguintes passos, utilizando o recíproco $\left(\mathrm{x}^{\wedge}-1\right)$, ou inverso multiplicativo, nos dados normalizados que, para este caso, substituem o IDH utilizado por Darela-Filho et al. (2016). Esse é um passo importante, pois torna os valores dos indicadores uma medida para a falta da capacidade adaptativa.

\section{Aplicação dos indicadores de vulnerabilidade no IVSC}

A análise estatística foi realizada a partir do software $R$, e utilizado o pacote "PACMAN". Foram selecionados os indicadores de vulnerabilidade (Tabela 1) e os dados foram preparados para a normalização. Os indicadores são normalizados para que os dados sejam compatíveis entre si, para isso, é atribuído a eles a mesma escala de valores. 
Desta forma, individualmente, para cada indicador, foi aplicada a seguinte fórmula de normalização:

$$
\text { Normalizado }=((\mathrm{Xi}-\mathrm{Xmin})) /((\mathrm{Xmax}-\mathrm{Xmin}))
$$

Onde, $X$ = representação do valor indicador de $\mathrm{i}, \mathrm{Xmin}=$ representação do valor mínimo encontrado na série, e Xman = representação do valor máximo encontrado na série. Ao utilizar essa fórmula, todos os dados foram transformados de forma que os valores de cada indicador variam de 0 a 1 .

Após esse processo os dados já normalizados, passam pelo reescalonamento, isso porque, cada indicador tem um efeito positivo ou negativo sobre a vulnerabilidade. Portanto, cada indicador é reescalonado com base em seu impacto direto ou indireto sobre a vulnerabilidade. Individualmente os indicadores foram avaliados quanto ao seu impacto, ou contribuição para a situação de vulnerabilidade. Atribuindo valores, quando um indicador tiver efeito positivo sobre a vulnerabilidade, quanto mais próximo de 1 for o valor obtido, maior seu impacto/peso para a vulnerabilidade. Os indicadores com impacto positivo na vulnerabilidade foram reescalonados utilizando a seguinte fórmula:

Dados positivos reescalonados $=$ Dados positivos $\times(1.0-0.1)+0.1$

Um indicador com impacto negativo na vulnerabilidade é reescalonado usando a seguinte fórmula:

Dados negativos reescalonados $=$ Dados negativos $\times(0.1-1.0)+1.0$

Por fim, após normalizados e reescalonados, foi calculada à agregação dos dados, agregando primeiramente as pontuações dos indivíduos em um único valor por indicador. A pontuação final é atribuída pela média geométrica dos indicadores.

\section{Resultados e discussão}

Darela Filho et al. (2016) elucidam que o Índice de Vulnerabilidade Socio-Climática (IVSC), adaptado a partir de Torres et al. (2012), é um índice que apresenta resultados espaciais, e que tem por principal finalidade indicar as regiões, através de uma base comparativa, que apresentam maior risco ou são mais vulneráveis aos extremos climáticos, oriundos das mudanças climáticas.

Os índices acima citados, foram adaptados para este estudo, e aqui não apresentam dados numéricos e não espaciais. Darela Filho et al. (2016) utilizam o IDH (Índice de Desenvolvimento Humano) como principal indicador social para compor a análise de vulnerabilidade em diferentes escalas geográficas. No caso do presente estudo, a utilização do IDH é substituída por variáveis adaptadas de um sistema de indicadores de vulnerabilidade climática criado especificamente por Gadelha et al. (2021), para pequenas comunidades rurais. 
Neste estudo, a vulnerabilidade de uma pequena comunidade rural pode ser mensurada através de uma variável numérica. A partir da utilização de indicadores locais, tornou-se possível aplicar o IVSC em uma pequena localidade. Ressalta-se que a confirmação dessa possibilidade, abre portas para que, através de metodologias adequadas, possam ser criados indicadores de vulnerabilidade que representem todo $o$ semiárido do estado da Paraíba e de outras regiões, dando meios para diversas formas de mapear as principais localidades mais vulneráveis aos extremos climáticos.

Seguindo o cálculo do IVSC, o resultado obtido para o município de Cabaceiras é IVSC $=5,60$. Esse resultado é o produto da multiplicação do valor obtido para a variável climática (IRMC $=13$ ), pela média geométrica do valor obtido para a variável social, que aqui será chamada de Indicadores de Vulnerabilidade Local (IVL $=0,43$ ), que são os indicadores propostos por Gadelha et al. (2021). Este cálculo adaptado, pode ser representado pela seguinte equação:

$$
\operatorname{IVS}_{j}=\mathrm{CI}_{j}^{*} \sqrt[n]{\prod_{i=1}^{n} I V L_{i j}}
$$

Nos resultados numéricos obtidos a partir desse cálculo, deve-se considerar que quanto maior o valor, maior o grau de vulnerabilidade climática local. Aponta-se que a população da zona rural do município de Cabaceiras é vulnerável aos extremos climáticos, não podendo ainda inferir comparações ou graus de vulnerabilidade. Considerando que o índice original parte da premissa de gerar resultados espaciais, não há como comparar valores em trabalhos anteriores, relacionando o IVSC resultados numéricos. A partir do resultado e da adaptação realizada neste estudo, espera-se criar uma base teórica, onde outros pesquisadores possam gerar resultados numéricos, para que seja realizada comparação de efetividade e representatividade do valor gerado, a partir do cálculo.

Quando se compara o valor do índice de vulnerabilidade obtido no presente estudo, com trabalhos quem tem escopo semelhante, como Combo et al. (2018), que ao investigar a vulnerabilidade climática de pequenos agricultores na Uganda, obteve o valor 10,0 para o condado mais vulnerável. Em sua maioria, os índices de vulnerabilidade possuem a sua classificação, dessa forma, o grau de vulnerabilidade proporcional ao aumento do valor obtido.

Muitos outros pesquisadores têm buscado simplificar, e até mesmo, popularizar os métodos que oferecem diagnóstico de vulnerabilidade climática para comunidades rurais, com foco em comunidades de agricultores familiares. Um exemplo, é o estudo de Chombo et al. (2018), que buscam adaptar e trazer novas metodologias que possibilitem a aplicação em pequenas regiões, e que possam sem adaptadas para outras escalas e locais do mundo. Da mesma forma que no presente estudo, priorizam a utilização de indicadores de vulnerabilidade que retratem a realidade local, podendo ser baseados em trabalhos anteriores, ou gerados a partir de coleta de dados em campo.

É importante deixar claro que, ao realizar um estudo sobre a vulnerabilidade no município de Cabaceiras, usando como base indicadores criados a partir da realidade local da zona rural do referido município, teve-se como principal intenção aprimorar o aspecto metodológico da análise de vulnerabilidade. Ao passo que foram escolhidos indicadores com informações pontuais que retratam a exposição, sensibilidade e capacidade adaptativa de agricultores da região, sendo considerados na adaptação do índice dados que retratam o homem do campo mediante a seca, ao invés de apenas dados demográficos.

Com o aumento das pesquisas que geram indicadores de vulnerabilidade climática, principalmente para localidades no estado da Paraíba, a exemplo de Nunes et al. (2020), é 
reforçado o apelo de que os instrumentos que são gerados no ambiente acadêmico nesta área específica, são cada dia mais pensados para a aplicação prática. Os estudos que priorizam ajustes metodológicos para pequenas localidades devem ser considerados importantes, uma vez que, tem potencial para serem instrumentos factuais no planejamento e gestão nas mais variadas esferas de governo, podendo colaborar efetivamente em ações de políticas públicas.

Em outros estudos que priorizam ajuste metodológico, o índice de vulnerabilidade é composto por análises separadas para o grau de exposição, sensibilidade e capacidade adaptativa, como é o caso de Chombo et al. (2018). 0 objetivo de que aqui fossem calculados conjuntamente indicadores que representassem todas as dimensões da vulnerabilidade climática, explicita a visão dos autores sobre a aplicabilidade do índice adaptado, e a possibilidade de ampliação das análises em estudos posteriores.

\section{Conclusão}

O Índice de Vulnerabilidade Socio-Climática (IVSC), da forma como foi adaptado e calculado neste estudo, oferece praticidade em sua execução, podendo ser facilmente aplicado para todo o estado da Paraíba e outras regiões do semiárido, desde que sejam utilizadas mais informações e mais indicadores que representem a realidade de um universo maior.

Os resultados desse trabalho evidenciam a necessidade cada vez maior de se observar os impactos das mudanças climáticas não só em amplas escalas, mas também de forma pontual, principalmente em regiões com municípios pequenos, e com comunidades rurais em condições de vulnerabilidade socioambiental. Sendo possível, diante de ajustes com a realidade local, contribuir com importantes e robustas informações para subsidiar ações concretas por parte do Governo Público, em todas as esferas.

\section{Conflito de interesses}

Os autores declaram não haver conflito de interesses.

\section{Referências}

Batavia, C.; Nelson, M. P. Translating climate change policy into forest management practice in a multiple-use context: The role of ethics. Climatic Change, v. 148, p. 81-94, 2018. https://doi.org/10.1007/s10584-018-2186-2

Beiser-Mcgrath, L. F.; Huber, R. A. Assessing the relative importance of phychological and demographic factors for predicting climate and environmental attitudes. Climate Change, v. 149, p. 335-347, 2018. https://doi.org/10.1007/s10584-018-2260-9

Butt, N.; Gallagher, R. Using species traits to guide conservation actions under climate change. Climatic Change, v. 151, p. 317-332, 2018. https://doi.org/10.1007/s10584-0182294-z

Cacioppo, J. T.; Reis, H. T.; Zautra, A. J. Social resilience: The value of social fitness with an application to the military. American Psychologist, v. 66, n. 1, p. 43-51, 2011. https://doi.org/10.1037/a0021419

Chombo, 0.; Lwasa, S.; Makoama, T. M. Spatial differentiation of small holder farmers' vulnerability to climate change in the Kyoga plains of Uganda. American Journal of Climate Change, v. 7, p. 624-648, 2018. https://doi.org/10.4236/ajcc.2018.74039 
Darela Filho, J. P.; Lapola, D. M.; Torres, R. R.; Lemos, M. C. Socio-climatic hotspots in Brazil: How changes driven by the new set of IPCC climatic projections affect their relevance for policy? Climatic Change, v. 136, p. 413-425, 2016. https://doi.org/10.1007/s10584-0161635-z

Gadelha, D. P.; Carvalho, T. K. N.; Lucena, R. F. P. Indicadores de vulnerabilidade climática, Etnobiologia e agricultores tradicionais: estudo de caso na Comunidade de São Francisco, no Cariri do Estado da Paraíba, Brasil. Revista Brasileira de Gestão Ambiental e Sustentabilidade, $\quad$ v. $8, \quad$ n. 18, p. 543-557, 2021. https://doi.org/10.21438/ rbgas(2021)081835

Giorgi, F. Climate change hot-spots. Geophysical Research Letters, v. 33, n. 8, L08707, 2006. https://doi.org/10.1029/2006GL025734

IBGE - Instituto Brasileiro de Geografia e Estatística. 2017. Disponível em: <http://cidades.ibge.gov.br/xtras/temas.php?lang=\&codmun=250310\&idtema=94\&searc $\mathrm{h}=$ paraiba|cabaceiras|censo-demografico-2010:-resultados-da-amostra-domicilios-->. Acesso em: 06 jul. 2017.

IPCC - Intergovernmental Panel on Climate Change. Summary for Policymakers. In: Stocker, T. F.; Qin, D.; Plattner, G.-K.; Tignor, M.; Allen, S. K.; Boschung, J.; Nauels, A.; Xia, Y.; Bex, V.; Midgley, P.M. (Eds.). Climate Change 2013: The Physical Science Basis. Contribution of Working Group I to the Fifth Assessment Report of the Intergovernmental Panel on Climate Change. Cambridge: Cambridge University Press, 2013.

Mainali, J.; Pricope, N. G. High-resolution spatial assessment of population vulnerability to the climate change in Nepal. Applied Geographic, v. 82, p.66-82, 2017. https://doi.org/10.1016/j.apgeog.2017.03.008

McCarthy, J. J.; Canziani, O. F.; Leary, N. A.; Dokken, D. J.; White, K. S. (Eds.). Climate change 2001: Impacts, adaptation and vulnerability. Cambridge: Cambridge University Press, 2001.

Nunes, S. H. P.; Dantas Neto, J.; Silva, P. F.; Santos, E. G.; Matos, R. M. Indicadores de vulnerabilidade da seca e avaliação da governança de 1991-2010 no semiárido Paraibano. Revista Ibero-Americana de Ciências Ambientais, v. 11, n. 3, p. 210-228, 2020. http://doi.org/10.6008/CBPC2179-6858.2020.003.0018

Torres, R. R.; Marengo, J. A. Climate change hotspots over South America: From CMIP3 to CMIP5 multimodel datasets. Theoretical and Applied Climatology, v. 117, p. 579-87, 2014. https://doi.org/10.1007/s00704-013-1030-x

Torres, R. R.; Lapola, D. M.; Marengo, J. A.; Lombardo, M. A. Socio-climatic hotspots in Brazil. Climatic Change, v. 115, p. 597-609, 2012. https://doi.org/10.1007/s10584-0120461-1

Informação da Licença: Este é um artigo Open Access distribuído sob os termos da Licença Creative Commons Attribution, que permite uso irrestrito, distribuição e reprodução em qualquer meio, desde que a obra original seja devidamente citada. 